Pacific Journal of Mathematics

WEIGHTED LATTICE PATH ray and David Paul Rosella 


\title{
WEIGHTED LATTICE PATHS
}

\author{
R. D. Fray and D. P. Roselle
}

A lattice path in the plane from $(0,0)$ to $(m, n)$ with weighted horizontal, vertical, and diagonal steps will be called a weighted lattice path. We determine the number of unrestricted weighted lattice paths, the number of paths below a line, and the number of paths which must remain between two parallel lines with unit slope. We also obtain generating functions for the number of paths which remain below the line $y=x$; these extend results obtained by Carlitz and Riordan for the ballot numbers.

The number, $\left(\begin{array}{c}m+n \\ n\end{array}\right)$, of unrestricted minimal lattice paths from $(0,0)$ to $(m, n)$ is an old and well-known result, see MacMahon [5, I, p. 167] or Feller [3, p. 68]. The Catalan number $c_{n}=\left(\begin{array}{c}2 n \\ n\end{array}\right) /(n+1)$ is the number of paths from $(0,0)$ to $(n, n)$ which never rise above the line $y=x$ (c.f. [1]). The enumeration of the paths which must remain below the line $y=a x+b$ has been obtained by Lyness [4], Mohanty and Narayana [7], and Carlitz, Roselle, and Scoville [2]. Recently several authors [6], [8], and [10] have considered the same problem when diagonal steps are allowed in addition to the usual horizontal and vertical steps, and Stanton and Cowan [11] studied the resulting numbers in a different connection.

In [6] Mohanty and Handa enumerate the unrestricted lattice paths from $(0,0)$ to $(m, n)$ where $\mu$ diagonal steps are allowed at each position. They also determine the number of such paths which must remain below the line $x=a y$, and they indicate that a formula can be obtained for the number of paths which must remain below $x=a y-b$, where $a$ and $b$ are nonnegative integers. All of these results have been extended to weighted lattice paths in the present paper.

We remark that we will treat the case of weighted lattice paths bounded above and below by functions which are not necessarily straight lines in a paper now under preparation. This paper will also contain a generalization of a problem posed by Elwyn Berlekamp and solved in [2].

2. Unrestricted lattice paths. By a weighted lattice path we mean a path from $(0,0)$ to $(n, k)$ where a lattice point $(i, j)$ may be approached from any of the lattice points $(i, j-1),(i-1, j)$, or $(i-1, j-1)$, and the horizontal steps are assigned the weight $x$, the 
vertical steps the weight $y$, and the diagonal steps the weight $z$. If $f(n, k)=f(n, k \mid x, y, z)$ denotes the number of weighted lattice paths from $(0,0)$ to $(n, k)$, then it is immediate that

$$
f(n, k)=x f(n-1, k)+y f(n, k-1)+z f(n-1, k-1),
$$

whenever $n, k \geqq 1$. It is clear that (2.1), together with the initial conditions $f(n, 0)=x^{n}$ and $f(0, k)=y^{k}$, determines $f(n, k)$. The following table gives the first few values of $f(n, k)$.

\begin{tabular}{|c|c|c|c|c|}
\hline$n$ & 0 & 1 & $\begin{array}{c}f(n, k) \\
2\end{array}$ & 3 \\
\hline 0 & 1 & $y$ & $y^{2}$ & $y^{3}$ \\
\hline 1 & $x$ & $2 x y+z$ & $3 x y^{2}+2 y z$ & $4 x y^{3}+3 y^{2} z$ \\
\hline 2 & $x^{2}$ & $3 x^{2} y+2 x z$ & $6 x^{2} y^{2}+6 x y z+z^{2}$ & $10 x^{2} y^{3}+12 x y^{2} z+3 y z^{2}$ \\
\hline 3 & $x^{3}$ & $4 x^{3} y+3 x^{2} z$ & $10 x^{3} y^{2}+12 x^{2} y z+3 x z^{2}$ & $20 x^{3} y^{3}+30 x^{2} y^{2} z+12 x y z^{2}+z^{3}$ \\
\hline 4 & $x^{4}$ & $5 x^{4} y+4 x^{3} z$ & $15 x^{4} y^{2}+20 x^{3} y z+6 x^{2} z^{2}$ & $35 x^{4} y^{3}+60 x^{3} y^{2} z+30 x^{2} y z^{2}+4 x z^{3}$ \\
\hline 5 & $x^{5}$ & $6 x^{5} y+5 x^{4} z$ & $21 x^{5} y^{2}+30 x^{4} y z+10 x^{3} z^{2}$ & $56 x^{5} y^{3}+105 x^{4} y^{2} z+60 x^{3} y z^{2}+10 x^{2} z^{3}$ \\
\hline
\end{tabular}

In the next place, if we put

$$
F_{k}(u)=\sum_{n=0}^{\infty} f(n, k) u^{n},
$$

it follows from (2.1) that

$$
F_{k}(u)=x u F_{k}(u)+y F_{k-1}(u)+z u F_{k-1}(u) .
$$

Repeated application of this recurrence gives

$$
\begin{aligned}
F_{k}(u) & =\left(\frac{y+z u}{1-x u}\right)^{k} F_{0}(u) \\
& =\left(\frac{y+z u}{1-x u}\right)^{k} \frac{1}{1-x u} \\
& =\sum_{n=0}^{\infty} u^{n} \sum_{r=0}^{n}\left(\begin{array}{l}
k \\
r
\end{array}\right)\left(\begin{array}{c}
n+k-r \\
k
\end{array}\right) x^{n-r} y^{k-r} z^{r} .
\end{aligned}
$$

Therefore we obtain

$$
f(n, k)=\sum_{r=0}^{n}\left(\begin{array}{l}
k \\
r
\end{array}\right)\left(\begin{array}{c}
n+k-r \\
k
\end{array}\right) x^{n-r} y^{k-r} z^{r} .
$$

In particular, we have

$$
\begin{gathered}
f(n, k \mid 1,1, z-1)=\sum_{r=0}^{n}\left(\begin{array}{l}
n \\
r
\end{array}\right)\left(\begin{array}{l}
k \\
r
\end{array}\right) z^{r}, \\
f(n, k \mid 1,1,0)=\left(\begin{array}{c}
n+k \\
k
\end{array}\right),
\end{gathered}
$$




$$
f(n, k \mid 1,1,1)=\sum_{r=0}^{n} \frac{(n+k-r) !}{r !(n-r) !(k-r) !},
$$

where (2.3) follows by an application of equation (5) on page 8 in [9]. The equation (2.4) is the familiar result stated in the introduction, and (2.5) is due to Moser and Zayachkowski [8] and Stanton and Cowan [11].

Note that it follows from (2.2) that

$$
f(k, n)=\left(\frac{y}{x}\right)^{n-k} f(n, k),
$$

and

$$
f(n, k \mid x, y, x y)=x^{n} y^{k} f(n, k \mid 1,1,1) .
$$

We remark that (2.2) could be obtained directly from equation (1) in [6]. It is also possible to give a simple combinatorial proof of (2.2). By a block on a weighted lattice path from $(0,0)$ to $(n, k)$, we mean one of the moves $(i, j) \rightarrow(i+1, j+1)$ or $(i, j) \rightarrow(i, j+1) \rightarrow$ $(i+1, j+1)$. Next notice that a path consists of blocks connected by moves of the form $(i, j) \rightarrow(i+h, j) \rightarrow(i+h, j+t)$. Now if a path contains $r$ blocks, it is immediate that the rows in which these blocks can occur can be chosen in $\left(\begin{array}{l}k \\ r\end{array}\right)$ ways and the columns may be chosen in $\left(\begin{array}{l}n \\ r\end{array}\right)$ ways. Since there are $n-r$ remaining horizontal steps and $k-r$ remaining vertical steps, and since a block has weight $x y+z$,

$$
\begin{aligned}
f(n, k) & =\sum_{r=0}^{n}\left(\begin{array}{l}
n \\
r
\end{array}\right)\left(\begin{array}{l}
k \\
r
\end{array}\right)(x y+z)^{r} x^{n-r} y^{k-r} \\
& =\sum_{s=0}^{n}\left(\begin{array}{l}
k \\
s
\end{array}\right)\left(\begin{array}{c}
n+k-s \\
k
\end{array}\right) x^{n-s} y^{k-s} z^{s} .
\end{aligned}
$$

In particular, it follows that

$$
f(n, k \mid x, y,-x y)=x^{n} y^{k} .
$$

3. Paths below $\mathrm{y}=\mathrm{x}+\mathbf{r}$ and $\mathrm{x}=\mathrm{sy}$. Next we obtain the number, $e(n, k, r \mid x, y, z)=e(n, k, r)$, of weighted lattice paths from $(0,0)$ to $(n, k)$ which remain below the line $y=x+r$, for $r$ a positive integer. Let $g(n, k, r)$ be the number of weighted paths from $(0,0)$ to $(n, k)$ which touch, cross, or traverse the line $y=x+r$. Then it is immediate that

$$
e(n, k, r)+g(n, k, r)=f(n, k) .
$$


First we shall determine $g(n, k, r)$ for ordinary lattice paths, that is, we let $x=y=1$ and $z=0$. Note that any such path $\alpha$ which touches $y=x+r$ does so at a last lattice point and that if the portion of $\alpha$ from this point to $(n, k)$ is reflected about the line $y=x+r$, we obtain a path $\alpha^{\prime}$ from $(0,0)$ to $(k-r, n+r)$. Since this procedure is reversible, we obtain

$$
g(n, k, r \mid 1,1,0)=\left(\begin{array}{l}
n+k \\
n+r
\end{array}\right) .
$$

In order to determine $g(n, k, r)$ for the general case, suppose we are given a weighted lattice path from $(0,0)$ to $(n, k)$ with $t$ diagonal steps which touches the line $y=x+r$. Removing the $t$ diagonal steps we obtain a path from $(0,0)$ to $(n-t, k-t)$ with no diagonal steps which touches $y=x+r$. Since we may reverse this process, we have from (3.2), allowing for the weighting factors,

$$
\left(\begin{array}{c}
n+k-2 t \\
n-t+r
\end{array}\right)\left(\begin{array}{c}
n+k-t \\
t
\end{array}\right) x^{n-t} y^{k-t} z^{t}=\left(\begin{array}{c}
k-r \\
t
\end{array}\right)\left(\begin{array}{c}
n+k-t \\
k-r
\end{array}\right) x^{n-t} y^{k-t} z^{t}
$$

paths from $(0,0)$ to $(n, k)$ with $t$ diagonal steps which touch $y=x+r$. Hence

$$
\begin{aligned}
g(n, k, r) & =\sum_{t=0}^{n+r}\left(\begin{array}{c}
k-r \\
t
\end{array}\right)\left(\begin{array}{c}
n+k-t \\
k-r
\end{array}\right) x^{n-t} y^{k-t} z^{t} \\
& =\left(\frac{y}{x}\right)^{r} f(n+r, k-r) .
\end{aligned}
$$

It follows from (3.1) that

$$
e(n, k, r)=f(n, k)-\left(\frac{y}{x}\right)^{r} f(n+r, k-r),
$$

where $n \geqq k-r$ and $1 \leqq r \leqq k$.

It will be convenient to let $b(n, k)$ denote the number of paths from $(0,0)$ to $(n, k)$ which remain below the line $y=x$. Since any such path must first go to $(1,0)$ we see that

$$
b(n, k)=x e(n-1, k, 1)=x f(n-1, k)-y f(n, k-1) .
$$

From this last expression and (2.2), we find that for $k \geqq 1$

$$
b(n, k)=\frac{n-k}{k} \sum_{r=0}^{n}\left(\begin{array}{l}
k \\
r
\end{array}\right)\left(\begin{array}{c}
n+k-1-r \\
k-1
\end{array}\right) x^{n-r} y^{k-r} z^{r} .
$$

In the next place, let $a(n, k)$ be the number of weighted paths from $(0,0)$ to $(n, k)$ which may touch, but not traverse or cross, the line $y=x$. Then for $n \geqq 1$, we have 


$$
b(n, k)=a(n, k)-y a(n, k-1) .
$$

This formula follows easily for $n=k$. For $n>k$ (3.4) will follow by showing that $y a(n, k-1)$ is the number of weighted lattice paths from $(0,0)$ to $(n, k)$ which must touch the line $y=x$ at a point different from $(0,0)$. Now a path which must touch $y=x$ does so at a last point, and the immediately preceding step must have been a vertical step. If this vertical step is removed, we obtain a path from $(0,0)$ to $(n, k-1)$ which may touch the line $y=x$. Since this process is reversible we obtain (3.4). Now from (3.3) and (3.4) we have

$$
\begin{aligned}
a(n, k) & =b(n, k)+y a(n, k-1) \\
& =\sum_{\jmath=0}^{k} y^{j} b(n, k-j) .
\end{aligned}
$$

After a little reduction we find that

$$
a(n, k)=\sum_{r=0}^{k} \frac{1}{n}\left(\begin{array}{l}
n \\
r
\end{array}\right) x^{n-r} y^{k-r} z^{r} \sum_{j=0}^{k-r}(n-r-j)\left(\begin{array}{c}
n+j-1 \\
j
\end{array}\right) .
$$

In particular,

$$
a(n, n \mid 1,1, z-1)=\sum_{r=0}^{n} \frac{1}{n}\left(\begin{array}{c}
n \\
r
\end{array}\right)\left(\begin{array}{c}
n \\
r+1
\end{array}\right) z^{r} .
$$

The numbers $a(n, n \mid 1,1,0)$ are the Catalan numbers mentioned in the introduction, and it is interesting to note that the coefficients of $z^{r}$ in the right member of (3.5) are what Riordan [9, p. 17] has called the Runyon numbers.

Let $s$ be a positive integer, and let $b(n, k, s)$ denote the number of weighted lattice paths from $(0,0)$ to $(n, k)$ which remain below the line $x=s y$. Then $b(s k, k, s)=0, b(n, 0, s)=x^{n}$, and

$$
\begin{aligned}
b(n, k, s)= & x b(n-1, k, s)+y b(n, k-1, s) \\
& +z b(n-1, k-1, s)
\end{aligned}
$$

whenever $n>s k$. It is now straightforward to verify that for $k \geqq 1$

$$
b(n, k, s)=\frac{n-s k}{k} \sum_{r=0}^{n}\left(\begin{array}{l}
k \\
r
\end{array}\right)\left(\begin{array}{c}
n+k-1-r \\
k-1
\end{array}\right) x^{n-r} y^{k-r} z^{r},
$$

which agrees with (3.3).

Again, if $a(n, k, s)$ denotes the number of weighted paths from $(0,0)$ to $(n, k)$ which may touch, but not traverse or cross, the line $x=s y$, then as before

$$
b(n, k, s)=a(n, k, s)-y a(n, k-1, s) .
$$


Combining this with (3.7) we have

$$
a(n, k, s)=\sum_{r=0}^{n} \frac{1}{n}\left(\begin{array}{l}
n \\
r
\end{array}\right) x^{n-r} y^{k-r} z^{r} \sum_{j=0}^{k-r}(n-s(r+j))\left(\begin{array}{c}
n+j-1 \\
j
\end{array}\right) .
$$

4. Paths bounded above and below. Let $D(n, k ; r, s)$ denote the number of ordinary lattice paths from $(0,0)$ to $(n, k)$ which remain below the line $y=x+r$ and above the line $y=x-s$, where $r$ and $s$ are positive integers. In order to determine $D(n, k ; r, s)$ we shall enumerate the paths from $(0,0)$ to $(n, k)$ with a particular touch sequence. A path from $(0,0)$ to $(n, k)$ is said to have a touch sequence of the form rsr, for example, if it touches or crosses the line $y=x+r$ one or more times without touching $y=x-s$, then touches $y=x-s$ several times without touching $y=x+r$, and then touches $y=x+r$. Note that if a path has a touch sequence of the form $r s r$, it also has sequences of the forms $r, s, r s$, and $s r$. We shall say that a path has a touch sequence of the form $r s h$ if it has a sequence of the form rsrs...rs with $h$ pairs $r s$, and it has a touch sequence of the form rrh if it has a sequence of the form $r s r s \cdots r s r$ with $h$ pairs $r s$. Similarly we define sequences of the form srh and ssh.

Let $\alpha$ be a path from $(0,0)$ to $(n, k)$ which possesses an $r s h$ touch sequence. In its last $r s h$ sequence, let $a_{1}$ be the last point where $\alpha$ touches $y=x+r$ in its first collection of $r$ touches; let $b_{1}$ be the last point where $\alpha$ touches $y=x-s$ in its first collection of $s$ touches; let $a_{2}$ be the last point where $\alpha$ touches $y=x+r$ in its second collection of $r$ touches, etc. Now reflect that portion of $\alpha$ between $\alpha_{1}$ and $(n, k)$ about the line $y=x+r$. This new path $\alpha^{\prime}$ goes from $(0,0)$ to $(k-r, n+r)$. The point $b_{1}$ now lies on the line $y=$ $x+2 r+s$. Reflect the portion of $\alpha^{\prime}$ from $b_{1}$ to $(k-r, n+r)$ about the line $y=x+2 r+s$. The new path $\alpha^{\prime \prime}$ goes from $(0,0)$ to $(n-r-s, k+r+s)$. Reflecting the portion of $\alpha^{\prime \prime}$ between $a_{2}$ (which now lies on $y=x+3 r+2 s)$ and $(n-r-s, k+r+s)$ about the line $y=x+3 r+2 s$ we obtain a new path from $(0,0)$ to

$$
(k-2 r-s, n+2 r+s) \text {. }
$$

Continuing this process we eventually obtain a path from $(0,0)$ to $(n-h(r+s), k+h(r+s))$. Since this process is reversible, the number of paths from $(0,0)$ to $(n, k)$ with an $r s h$ touch sequence is $\left(\begin{array}{c}n+k \\ k+h(r+s)\end{array}\right)$. Similarly, the number of paths with an rrh touch sequence is $\left(\begin{array}{c}n+k \\ k-r-h(r+s)\end{array}\right)$, the number with an srh touch sequence is $\left(\begin{array}{c}n+k \\ k-h(r+s)\end{array}\right)$; and the number with an ssh touch sequence is 
$\left(\begin{array}{c}n+k \\ k+s+h(r+s)\end{array}\right)$. Hence we have that

$$
\begin{aligned}
D(n, k ; r, s)=\left(\begin{array}{c}
n+k \\
k
\end{array}\right) & -\sum_{h=0}\left(\begin{array}{c}
n+k \\
k-r-h(r+s)
\end{array}\right) \\
& -\sum_{h=0}\left(\begin{array}{c}
n+k \\
k+s+h(r+s)
\end{array}\right) \\
& +\sum_{h=1}\left(\begin{array}{c}
n+k \\
k+h(r+s)
\end{array}\right) \\
& +\sum_{h=1}\left(\begin{array}{c}
n+k \\
k-h(r+s)
\end{array}\right) .
\end{aligned}
$$

If $\alpha$ is a weighted lattice path from $(0,0)$ to $(n, k)$ with $t$ diagonal steps having an $r s h$ touch sequence, then by romoving the diagonal steps we obtain a path from $(0,0)$ to $(n-t, k-t)$ with an rsh touch sequence. Hence the number of weighted paths from $(0,0)$ to $(n, k)$ with $t$ diagonal steps having an $r s h$ touch sequence is

$$
\left(\begin{array}{c}
n+k-2 t \\
k-t+h(r+s)
\end{array}\right)\left(\begin{array}{c}
n+k-t \\
t
\end{array}\right) x^{n-t} y^{k-t} z^{t}
$$

Therefore the number of weighted paths from $(0,0)$ to $(n, k)$ with an $r s h$ touch sequence is

$$
\begin{aligned}
& \sum_{t=0}\left(\begin{array}{c}
k+h(r+s) \\
t
\end{array}\right)\left(\begin{array}{c}
n+k-t \\
k+h(r+s)
\end{array}\right) x^{n-t} y^{k-t} z^{t} \\
= & \left(\frac{y}{x}\right)^{-h(r+s)} f(n-h(r+s), k+h(r+s)) .
\end{aligned}
$$

Similarly, the number of weighted paths with an rrh touch sequence is $(y / x)^{r+h(r+s)} f(n+r+h(r+s), k-r-h(r+s))$, the number with an srh touch sequence is $(y / x)^{h(r+s)} f(n+h(r+s), k-h(r+s))$, and the number with an ssh touch sequence is

$$
(y / x)^{-s-h(r+s)} f(n-s-h(r+s), k+s+h(r+s)) .
$$

Hence if $d(n, k ; r, s)$ is the number of weighted lattice paths from $(0,0)$ to $(n, k)$ which remain below the line $y=x+r$ and above the line $y=x-s$, then

$$
\begin{aligned}
d(n, k ; r, s)= & f(n, k) \\
& -\sum_{h=0}\left(\frac{y}{x}\right)^{r+h(r+s)} f(n+r+h(r+s), k-r-h(r+s)) \\
& -\sum_{h=0}\left(\frac{y}{x}\right)^{-s-h(r+s)} f(n-s-h(r+s), k+s+h(r+s))
\end{aligned}
$$




$$
\begin{aligned}
& +\sum_{h=1}\left(\frac{y}{x}\right)^{-h(r+s)} f(n-h(r+s), k+h(r+s)) \\
& +\sum_{h=1}\left(\frac{y}{x}\right)^{h(r+s)} f(n+h(r+s), k-h(r+s)) .
\end{aligned}
$$

5. The number of paths below $\mathrm{y}=\mathrm{sx}+\mathrm{r}$. Let $e(n, k ; r, s)$ be the number of weighted lattice paths from $(0,0)$ to $(n, k)$ which remain below the line $x=s y-r$, where $s$ and $r$ are integers, $s \geqq 1$ and $r \geqq 0$. Also let $r=s q-t$ where $0 \leqq t<s$. Since

$$
e(n, k ; r, s)=f(n, k)
$$

if $k<q$ or $n<t$, we shall assume throughout this section that $k \geqq q$ and $n \geqq t$. As before we shall determine the number of paths from $(0,0)$ to $(n, k)$ which touch the line $x=s y-r$. Any such path must touch the line at a last point, which will have coordinates $(t+s j, q+j)$ for $j=0,1, \cdots, k-q$. The number of paths from $(0,0)$ to $(n, k)$ which touch $x=s y-r$ for the last time at $(t+s j, q+j)$ is $f(t+s j, q+j) b(n-t-s j, k-q-j, s)$. Thus the total number of paths from $(0,0)$ to $(n, k)$ which touch $x=s y-r$ is

$$
\sum_{j=0}^{k-q} f(t+s j, q+j) b(n-t-s j, k-q-j, s) .
$$

Then

$$
\begin{aligned}
e(m+t, p+q ; r, s)= & f(m+t, p+q) \\
& -\sum_{j=0}^{p} f(t+s j, q+j) b(m-s j, p-j, s)
\end{aligned}
$$

for any nonnegative integers $m$ and $p$. Combining (5.1) with (2.2) and (3.7) we have that the second term on the right of (5.2) has the explicit expression

$$
\begin{gathered}
\sum_{u=0}^{p+q} x^{m+t-u} y^{p+q-u} z^{u} \sum_{j=0}^{p} \sum_{h=0}^{u} \frac{m-s p}{p-j}\left(\begin{array}{c}
q+j \\
u-h
\end{array}\right)\left(\begin{array}{c}
p-j \\
h
\end{array}\right) \\
\left(\begin{array}{c}
q+t+j(s+1)-u+h \\
q+j
\end{array}\right)\left(\begin{array}{c}
m+p-j(s+1)-1-h \\
p-j-1
\end{array}\right) .
\end{gathered}
$$

The numbers $e(n, k ; r, s)$ can also be expressed as a determinant. Counting the number of paths from $(0,0)$ to $(n+r, k)$ which remain below $x=s y$ according to the points where these paths intersect the line $x=r$, we have

$$
b(n+r, k, s)=x^{r} e(n, k ; r, s)+\sum_{j=1}^{q-1} h(r, j, s) e(n, k-j ; r-j s, s),
$$

where 


$$
h(r, j, s)=b(r, j, s)-b(r, j-1, s) .
$$

By repeated application of (5.3) we get

$$
e(n, k ; r, s)=x^{-r} \sum_{j=0}^{q-1}(-1)^{j} C(r, s, j) b(n+r-j s, k-j, s),
$$

where $C(r, s, 0)=1$ and, for $j \geqq 1, C(r, s, j)$ is the determinant of the $j \times j$ matrix $\left(c_{u, v}\right)$ defined by

$$
c_{u, v}= \begin{cases}0 & \text { if } v>u+1 \\ 1 & \text { if } v=u+1 \\ x^{u s-r} h(r-(v-1) s, u-v+1, s) & \text { if } v<u+1\end{cases}
$$

In order to prove (5.4) note that if $C(r, s, j)$ is expanded about its first column, we have

$$
C(r, s, j)=\sum_{i=1}^{j}(-1)^{i+1} x^{i s-r} h(r, i, s) C(r-i s, s, j-i) .
$$

The proof of (5.4) will be completed by induction on $q$. For $q=1$ the equation follows directly from (5.3). Assuming the equation true for integers less than or equal to $q$ we have from (5.3) and (5.4) that

$$
\begin{aligned}
e(n, k ; r, s)= & x^{-r} b(n+r, k, s) \\
& -x^{-r} \sum_{j=1}^{q} h(r, j, s) x^{-(r-j s)} \\
& \times \sum_{i=0}^{q-j}(-1)^{i} C(r-j s, s, i) b(n+r-s(i+j), k-i-j, s) \\
= & x^{-r} b(n+r, k, s) \\
& -x^{-r} \sum_{u=1}^{q} b(n+r-u s, k-u, s) \\
& \times \sum_{j=1}^{u}(-1)^{u-j} x^{-(r-j s)} h(r, j, s) C(r-j s, s, u-j) .
\end{aligned}
$$

We then obtain the desired result by applying (5.5).

The determinantal expression for $e(n, k ; r, s)$ follows from (5.4); specifically,

$$
e(n, k ; r, s)=(-1)^{q-1} \operatorname{det}\left(v_{i j}\right)
$$

where $\left(v_{i j}\right)$ is the $q \times q$ matrix defined by

$$
v_{i j}= \begin{cases}0 & \text { if } j>i+1 \\ 1 & \text { if } j=i+1 \\ x^{-r} b(n+r-(j-1) s, k-j+1, s) & \text { if } i=q \\ x^{i s-r} h(r-(j-1) s, i-j+1, s) & \text { if } j \leqq i<q .\end{cases}
$$


Now let $\alpha$ be any path from $(0,0)$ to $(n, k)$ which remains below the line $y=s x+r$, where $s$ is a positive integer. If we interchange the initial and terminal points of $\alpha$ and reverse the direction of the path, we obtain a path from $(0,0)$ to $(k, n)$ which remains below the line $x=s y-n s+k-r$ and whose horizontal and vertical weights have been interchanged. Since this procedure gives us a one-to-one correspondence, we have that the number of paths from $(0,0)$ to $(n, k)$ which remain below $y=s x+r$, for $s$ a positive integer, is $(y / x)^{-(n-k)}$ $e(k, n ; n s+r-k, s)$.

\section{Generating functions for $b(n, k)$. Let}

$$
b_{n}(u)=\sum_{k=0}^{n-1} b(n, k) u^{k} .
$$

Since the numbers $b(n, k)$ satisfy the recurrence (3.6) with $s=1$, we obtain

$$
(1-y u) b_{n}(u)=(x+z u) b_{n-1}(u)-y u^{n} b(n, n-1)
$$

for $n>1$. Repeated application of this relation yields

$$
(1-y u)^{n} b_{n}(u)=x(x+z u)^{n-1}-y \sum_{j=1}^{n}(1-y u)^{j-1}(x+z u)^{n-j} u^{j} b(j, j-1),
$$

which is an extension of equation (2.3) in [1].

Next we shall determine the generating function $b(u)$ for $b(n+1, n)$. By definition $b(n+1, n)$ is the number of weighted lattice paths from $(0,0)$ to $(n+1, n)$ which remain below $y=x$. We shall count these paths according to the last point, excluding $(n+1, n)$, where each touches $y=x-1$. There are $y b(1,0) b(n, n-1)$ paths which last touch $y=x-1$ at $(1,0)$; there are $y b(2,1) \times$ $b(n-1, n-2)$ paths which last touch the line at $(2,1)$, etc. Hence

$$
b(n+1, n)=z b(n, n-1)+y \sum_{j=0}^{n-1} b(j+1, j) b(n-j, n-1-j)
$$

for $n \geqq 1$. Then

$$
\begin{aligned}
b(u) & =\sum_{n=0}^{\infty} b(n+1, n) u^{n} \\
& =x+z u b(u)+y u b^{2}(u),
\end{aligned}
$$

and therefore

$$
b(u)=\frac{1}{2 y u}\left[(1-u z)-\sqrt{(1-u z)^{2}-4 x y u}\right] .
$$

For $x=y=1$ and $z=0$ this reduces to the generating function for 
the Catalan numbers (c.f. equation (1.3) in [1]).

We can also determine the generating functions

$$
\beta_{k}(u)=\sum_{n=k+1}^{\infty} b(n, k) u^{n}
$$

and

$$
b(u, v)=\sum_{n=1}^{\infty} \sum_{k=0}^{n-1} b(n, k) u^{n} v^{k}
$$

Note that

$$
b(u, v)=\sum_{n=1}^{\infty} b_{n}(v) u^{n}=\sum_{k=0}^{\infty} \beta_{k}(u) v^{k} .
$$

From (6.1) it follows that

$$
(1-y v) b(u, v)=x u+(x+z v) u b(u, v)-y u v b(u v),
$$

and hence

$$
\begin{aligned}
b(u, v)= & u(x-y v b(u v))[(1-x u)-v(y+z u)]^{-1} \\
= & u(x-y v b(u v)) \sum_{k=0}^{\infty} v^{k} \sum_{n=0}^{\infty} f(n, k) u^{n} \\
= & \sum_{k=0}^{\infty} \sum_{n=0}^{\infty} x f(n, k) v^{k} u^{n+1} \\
& -\sum_{k=0}^{\infty} \sum_{n=0}^{\infty} \sum_{s=0}^{\infty} y f(n, k) b(s+1, s) v^{k+s+1} u^{n+s+1} .
\end{aligned}
$$

Therefore

$$
\begin{aligned}
\beta_{k}(u) & =\sum_{n=0}^{\infty} x f(n, k) u^{n+1}-\sum_{n=0}^{\infty} u^{n+1} \sum_{s=0}^{k-1} y f(n-s, k-1-s) b(s+1, s) \\
& =\frac{u(y+z u)^{k}}{\left(1-x v^{k}\right)^{k+1}}\left\{x-y \sum_{s=0}^{k-1} b(s+1, s)\left(\frac{1-x u}{y+z u}\right)^{s+1} u^{s}\right\} .
\end{aligned}
$$

The equations (6.2) and (6.3) are extensions of equations (3.1) and (3.10) in [1]. Comparing coefficients of $u$ in (6.3) we have for $n<k$ that

$$
f(n, k)=\frac{y}{x} \sum_{s=0}^{n} f(n-s, k-1-s) b(s+1, s),
$$

and for $n>k$

$$
f(n, k+1)=\frac{1}{x}\left[b(n+1, k+1)+y \sum_{s=0}^{k} f(n-s, k-s) b(s+1, s)\right] .
$$

Of course these expressions can be obtained directly; this would give another derivation of (6.3). 


\section{REFERENCES}

1. L. Carlitz and J. Riordan, Two element lattice permutation numbers and their q-generalizations, Duke Math. J., 31 (1964), 371-388.

2. L. Carlitz, D. P. Roselle, and R. A. Scoville, Some remarks on Catalan-type sequences of positive integers, to appear in J. Combinatorial Theory.

3. William Feller, An Introduction to Probability Theory and Its Applications, 3rd ed., Wiley, New York, 1968.

4. R. C. Lyness, Al Capone and the death ray, Math. Gaz., 25 (1941), 283-287.

5. P. A. MacMahon, Combinatory Analysis, 2 vols. in 1, New York, Chelsea, 1960; originally published in 1915 and 1916.

6. S. G. Mohanty and B. R. Handa, On lattice paths with several diagonal sieps, Canadian Math. Bull., 11 (1968), 537-545.

7. S. G. Mohanty and T. V. Narayana, Some properties of compositions and their applications to probability and statistics I, Biometrische Z., 3 (1961), 252-258.

8. L. Moser and W. Zayachkowski, Lattice paths with diagonal steps, Scripta Math., 26 (1961), 223-229.

9. John Riordan, Combinatorial Identities, Wiley, New York, 1968.

10. V. K. Rohatgi, A note on lattice paths with diagonal steps, Canadian Math. Bull., $\boldsymbol{7}(1964), 470-472$.

11. R. G. Stanton and D. D. Cowan, Note on a "square" functional equation, SIAM Review, 12 (1970), 277-279.

Received July 23, 1970. The first author was supported in part by a summer grant from the Florida State University Committee on Faculty Research Support. The second author was supported in part by National Science Foundation grant GP-11397.

Florida State University and

Louisiana State UnIVERsity 


\section{PACIFIC JOURNAL OF MATHEMATICS}

\section{EDITORS}

H. SAMELSON

Stanford University

Stanford, California 94305

C. R. Новву

University of Washington

Seattle, Washington 98105
J. DUGundJI

Department of Mathematics

University of Southern California

Los Angeles, California 90007

RICHARD ARENS

University of California

Los Angeles, California 90024

\section{ASSOCIATE EDITORS}
E. F. BECKENBACH
B. H. NeumanN
F. WOLF
K. YOSHIDA

\section{SUPPORTING INSTITUTIONS}
UNIVERSITY OF BRITISH COLUMBIA
CALIFORNIA INSTITUTE OF TECHNOLOGY
UNIVERSITY OF CALIFORNIA
MONTANA STATE UNIVERSITY
UNIVERSITY OF NEVADA
NEW MEXICO STATE UNIVERSITY
OREGON STATE UNIVERSITY
UNIVERSITY OF OREGON
OSAKA UNIVERSITY
UNIVERSITY OF SOUTHERN CALIFORNIA
STANFORD UNIVERSITY
UNIVERSITY OF TOKYO
UNIVERSITY OF UTAH
WASHINGTON STATE UNIVERSITY
UNIVERSITY OF WASHINGTON
AMERICAN MATHEMATICAL SOCIETY CHEVRON RESEARCH CORPORATION TRW SYSTEMS
NAVAL WEAPONS CENTER 


\section{Pacific Journal of Mathematics}

\section{Vol. 37, No. $1 \quad$ January, 1971}

Gregory Frank Bachelis and Haskell Paul Rosenthal, On unconditionally

converging series and biorthogonal systems in a Banach space .........

Richard William Beals, On spectral theory and scattering for elliptic

operators with singular potentials .........................

J. Lennart (John) Berggren, Solvable and supersolvable groups in which every element is conjugate to its inverse ........................ 21

Lindsay Nathan Childs, On covering spaces and Galois extensions ..........

William Jay Davis, David William Dean and Ivan Singer, Multipliers and

unconditional convergence of biorthogonal expansions..............

Leroy John Derr, Triangular matrices with the isoclinal property ............

Paul Erdős, Robert James McEliece and Herbert Taylor, Ramsey bounds for

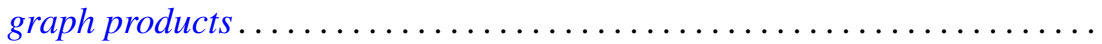

Edward Graham Evans, Jr., On epimorphisms to finitely generated

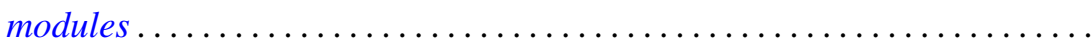

Hector O. Fattorini, The abstract Goursat problem ................. 51

Robert Dutton Fray and David Paul Roselle, Weighted lattice paths .........

Thomas L. Goulding and Augusto H. Ortiz, Structure of semiprime $(p, q)$

radicals ...........................................

E. W. Johnson and J. P. Lediaev, Structure of Noether lattices with join-principal maximal elements ....

David Samuel Kinderlehrer, The regularity of minimal surfaces defined over

slit domains

Alistair H. Lachlan, The transcendental rank of a theory. .

Frank David Lesley, Differentiability of minimal surfaces at the boundary ...

Wolfgang Liebert, Characterization of the endomorphism rings of divisible torsion modules and reduced complete torsion-free modules over complete discrete valuation rings....

Lawrence Carlton Moore, Strictly increasing Riesz norms.

Raymond Moos Redheffer, An inequality for the Hilbert transform ...

James Ted Rogers Jr., Mapping solenoids onto strongly self-entwined,

circle-like continua..........................

Sherman K. Stein, B-sets and planar maps ................... 217

Darrell R. Turnidge, Torsion theories and rings of quotients of Morita

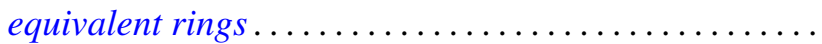

Fred Ustina, The Hausdorff means of double Fourier series and the principle of localization ................................

Stanley Joseph Wertheimer, Quasi-compactness and decompositions for arbitrary relations.

Howard Henry Wicke and John Mays Worrell Jr., On the open continuous images of paracompact $\check{C}$ ech complete spaces... 